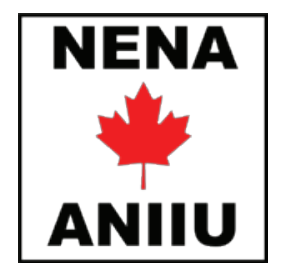

CANADIAN JOURNAL of EMERGENCY NURSING

JOURNAL CANADIEN des INFIRMIÈRES D'URGENCE

THE OFFICIAL JOURNAL OF THE NATIONAL EMERGENCY NURSES' ASSOCIATION

WWW.NENA.ca

WWw.CJEN.ca

\title{
What's in a BP:
}

\section{Do you FOLLOW THE MAP?}

\author{
Jennifer Davis', BN, BSc, RN, and Dawn Peta², BN, RN, ENC(C) \\ 'Emergency Nurse, Alberta Health Services \\ ${ }^{2}$ Clinical Educator Rural South West Zone, Alberta Health Services
}

\begin{abstract}
The purpose of this case study is to review a case of a patient in the emergency department with a complaint of acute on chronic back pain and shortness of breath. We propose that monitoring and trending blood pressure, mean arterial pressure and pulse pressure in patients who present with symptoms that may indicate a pulmonary and/or cardiac complaint, along with abnormal vital signs or abnormal lab values, would result in more timely intervention. Through this case study, we hope to encourage healthcare professionals to consider the importance of trending mean arterial pressure on helping to identify early hypovolemic shock, severe sepsis and other significant life-threatening conditions, as well as the need for continued nursing education.
\end{abstract}

Initial patient presentation and relevant history I $\begin{aligned} & \text { r. B was a } 60 \text {-year-old male who presented to the } \\ & \text { Emergency Department (ED) of a Level Three trauma }\end{aligned}$ centre at 2200 . His chief complaint at triage was shortness of breath and non-specific back pain. He stated that he had a history of chronic back pain from a work-related injury; however, this was "different pain". The vital signs at triage are presented in Table 1. Mr. B denied other significant medical conditions, but 25 pack-year smoking history. He was given a Canadian Triage and Acuity Scale (CTAS) score of 3 and sent to the waiting room.

\section{Relevant examination and diagnostic findings}

Mr. B was assessed by the emergency nurse and physician approximately one-hour post-arrival to the ED. The differential diagnoses considered by the ED physician included myocardial infarction (MI) and pulmonary embolism (PE). Blood work, a urinalysis and a chest $\mathrm{x}$-ray were ordered, along with an intravenous (IV) bolus of two litres of normal saline due to tachycardia and hypotension. At $0100 \mathrm{hrs}$, his troponin was reported to be $0.1 \mathrm{ng} / \mathrm{L}$; a repeat troponin and electrolyte panel was ordered for 0500. Refer to Table 2 for relevant laboratory results. His chest $\mathrm{x}$-ray was normal, and his urinalysis was unremarkable. A 12-lead electrocardiogram tracing showed sinus tachycardia with no acute ischemic or injury changes. Mr. B remained hypotensive after the two litres of normal saline.

\begin{tabular}{|l|l|}
\hline \multicolumn{2}{|l|}{ Table 1. Vital signs at triage } \\
\hline $\begin{array}{l}\text { Vital } \\
\text { signs: }\end{array}$ & $\begin{array}{l}\text { Blood Pressure (BP) } 95 / 64 \text { millimeters of mercury } \\
\text { (mmHg) } \\
\text { - Heart Rate (HR) } 126 \text { beats per minute (bpm) } \\
\text { - Temperature (T) } 36.9 \text { Celsius } \\
\text { - Respiratory Rate (RR) } 18 \\
\text { - Oxygen Saturation (Sp02) 93\% on room air. }\end{array}$ \\
\hline
\end{tabular}

Table 2. Relevant Laboratory Results

\begin{tabular}{|l|l|l|l|}
\hline Lab Test & $\begin{array}{l}\text { Patient } \\
\text { result } \\
\mathbf{0 1 0 0 h}\end{array}$ & $\begin{array}{l}\text { Patient } \\
\text { result } \\
\mathbf{0 7 0 0 h}\end{array}$ & Normal value \\
\hline $\begin{array}{l}\text { White } \\
\text { blood cell } \\
\text { count }\end{array}$ & 14.6 & $\begin{array}{l}\text { Repeat not } \\
\text { ordered }\end{array}$ & $4.5-11.0 \times 10^{3} / \mathrm{mm}^{3}$ \\
\hline Troponin I & 0.10 & 0.10 & $<0.02 \mathrm{ng} / \mathrm{L}$ \\
\hline $\begin{array}{l}\text { Serum } \\
\text { creatinine }\end{array}$ & 218 & 265 & $60-110 \mathrm{mmol} / \mathrm{L}$ \\
\hline Lactate & 2.5 & 2.8 & $<2 \mathrm{mmol} / \mathrm{L}$ \\
\hline D-dimer & 1662 & $\begin{array}{l}\text { Repeat not } \\
\text { ordered }\end{array}$ & $<500 \mathrm{ng} / \mathrm{ml}$ \\
\hline
\end{tabular}


Given the results of the diagnostic tests, including an elevated D-dimer with shortness of breath and back pain, the physician considered that these may indicate a PE. At this hospital site, there is no specialized on-site diagnostic imaging available during the night. As such, the physician prescribed a weightbased dose of low molecular weight heparin (LMWH), IV crystalloid, and for Mr. B to remain in the ED for a ventilation/ perfusion (VQ) scan in the morning. Mr. B was placed on Q 30 minute automatic BP and HR monitoring, provided with a call bell, and was advised to try to sleep. Refer to Table 3 for Mr. B's vital signs during the night.

While the day shift staff nurse completed the morning assessment, Mr. B reported increased back pain and shortness of breath; he also requested analgesia. Repeat vital signs at 0712 were a BP $83 / 40 \mathrm{mmHg}$ and $\mathrm{HR}$ of $127 \mathrm{bpm}$. The nurse assured Mr. B that his pain would be discussed with the physician; the

\section{Table 3. Blood pressure and heart rate recordings}

\begin{tabular}{|l|c|c|}
\hline Time & BP (MAP) & Heart rate (HR) \\
\hline 0112 & $88 / 53(64)$ & 115 \\
\hline 0142 & No BP recorded & No HR recorded \\
\hline 0212 & $106 / 47(66)$ & 104 \\
\hline 0242 & No BP recorded & No HR recorded \\
\hline 0312 & $120 / 89(98)$ & 112 \\
\hline 0342 & $76 / 42(52)$ & 121 \\
\hline 0412 & $89 / 43(57)$ & 118 \\
\hline 0442 & $95 / 63(72)$ & 118 \\
\hline 0512 & $93 / 58(69)$ & 121 \\
\hline 0542 & $88 / 53(64)$ & 125 \\
\hline 0612 & $99 / 47(64)$ & 123 \\
\hline 0642 & $110 / 44(65)$ & 126 \\
\hline 0712 & $83 / 40(53)$ & 127 \\
\hline
\end{tabular}

nurse was hesitant to administer analgesia due to his low BP. Mr. B's repeat blood work reported the troponin unchanged and his creatinine increased.

\section{Case Progression}

Throughout the morning, Mr. B became increasingly agitated; he began pacing the room and reiterated that his back hurt too much for him to lay still. Given the complaint of increased back pain, the nurse determined the need to obtain bilateral BPs as she was concerned that Mr. B could have an abdominal aortic aneurysm (AAA). The nurse attempted to obtain bilateral BPs with both automatic and manual cuffs; however, there was a lack of measurable BP in Mr. B's left arm. The nurses also repeated the bilateral BPs while Mr. B was lying supine; his left arm pressure remained absent and his right arm BP was 131/30mmHg. The nurse was concerned about the lack of BP in the left arm and the widening pulse pressure; the day shift ED physician was immediately notified. Upon reassessment, the day shift physician decided to order an urgent chest and abdomen $\mathrm{CT}$ to rule out $\mathrm{AAA}$; the radiologist confirmed that Mr. B had a ruptured AAA.

\section{Case outcome}

As Mr. B was at a Level Three trauma and surgical centre, the ED physician made arrangements for aeromedical transfer to a designated vascular surgery site approximately 200 kilometres north of the current location. The nursing staff quickly prepared Mr. B for transfer, established additional IV access, administered a dose of protamine sulphate to reverse the effects of the LMWH and monitored his condition closely. Mr. B's status continued to deteriorate as he waited for transfer, with signs and symptoms of hypovolemic shock. His back pain increased to 7/10 and he began to vomit; anti-emetics were given, but narcotics were held due to hypotension. By the time the aeromedical crew arrived, Mr. B had become increasingly hemodynamically unstable and his transfer was slightly delayed on the helicopter pad until the crew was certain he was safe to move.

Mr. B arrived at the designated hospital for vascular surgery in a pre-arrest condition, showing signs of decompensated shock, with a declining level of consciousness and a HR of $160 \mathrm{bpm}$. He was taken for emergent surgery where an aortic graft was completed. He survived surgery and was taken to intensive care, where it was determined he had acute kidney injury and emergency hemodialysis was commenced. During his first night in the intensive care unit, Mr. B succumbed to his critical condition and died.

\section{Case discussion}

Abdominal aortic aneurysm rupture is a life-threatening condition that requires rapid diagnosis, emergency resuscitation and surgical intervention. The Centers for Disease Control and Prevention (2020) report that ruptured AAA has an overall mortality rate of $80 \%$ and was the primary cause of death in over 9,923 patients in the United States in 2018. A leaking AAA may show no signs or symptoms until it has expanded or ruptured; the patient may present with an acute abdomen or back pain (Badger et al., 2014). A patient with a ruptured AAA may present with acute chest pain indicating a ripping or tearing sensation, rapid or weak thready peripheral pulses, dyspnea, nausea shortness of breath, inability to speak, weakness or paralysis on one side of the body, back pain, loss of vision or even loss of consciousness (Woodrow, 2011). When Mr. B was told his diagnosis, he then recalled a strong family history of AAA. Refer to Table 4 for AAA symptoms and risk factors.

Upon reviewing the details of Mr. B's case, we determined that there were several factors that indicated he was suffering from a ruptured AAA, as well as a number of factors that may have impeded the recognition of his condition. Confounding factors include dyspnea (acute on chronic given history of smoking), a history of chronic back pain and weakly elevated troponin values. We believe that Mr. B's fluctuating BP, subsequent hypovolemic shock, increased serum creatinine and significantly 
Table 4. Symptomology and Risk Factors for AAA

\begin{tabular}{|l|l|l|}
\hline Common signs \& symptoms & Risk ractors for Mr. B & Mr. B's signs \& symptoms \\
\hline $\begin{array}{l}\text { Deep constant pain in abdomen can indicate } \\
\text { enlarging aneurysm. Sudden, intense persistent } \\
\text { back or abdominal pain can indicate rupture. }\end{array}$ & Tobacco use Was a 25+ year smoker & $\begin{array}{l}\text { Back pain stated different from his } \\
\text { chronic back pain }\end{array}$ \\
\hline $\begin{array}{l}\text { Pulsating near belly button can indicate } \\
\text { enlargement }\end{array}$ & Being male & Not documented \\
\hline Low blood pressure & Being white & Hypotensive initial BP 95/64 mmHg \\
\hline Tachycardia & $\begin{array}{l}\text { Mr. B discloses a positive family } \\
\text { history }\end{array}$ & Tachycardia $126 \mathrm{bpm}$ \\
\hline Weak or thready pulse & Atherosclerosis & Not documented \\
\hline Shortness of breath & Known hypertension & Short of breath \\
\hline Signs of shock & Age $>65$ & $\begin{array}{l}\text { Initial VS could be classified as early } \\
\text { signs of shock }\end{array}$ \\
\hline
\end{tabular}

elevated D-dimer were indicators of Mr. B's acute condition, and that closer evaluation of these could have led to an earlier diagnosis of ruptured AAA.

\section{The importance of trending BP, MAP and PP}

When Mr. B's medical record was reviewed, a wide variation in his BP measurements throughout his seven-hour stay in the ED were noted (refer to Table 3 for vital signs). Scheetz (2006) presented a case report of an acute aortic dissection that also noted extreme fluctuations in BP with pressures that ranged from hypotensive with a systolic pressure under $75 \mathrm{mmHg}$ and hypertensive with diastolic pressures greater than $100 \mathrm{mmHg}$. MercerDeadman (2014) notes that the presentation of aortic aneurysm and rupture are often similar as hypotension in the dissected patient is often profound. At different times during his emergency room stay, Mr. B also experienced hypotension (refer to Table 3). Mercer-Deadman notes that while both dissection and rupture are uncommon, the latter is quite rare and is often difficult to diagnose in the ED. The rarity of these conditions, as well as their shared risk factors and similar presentations, can often cause confusion amongst practitioners assessing and recognizing dissections and rupture. Refer to Table 4 for the differences between aortic dissection and AAA rupture. Furthermore, these conditions also mimic other common conditions seen in the ED. Upon review of Scheetz's (2006) case, we believe that monitoring and evaluation of Mr. B's mean arterial pressure (MAP) may have led to an earlier diagnosis.

Mean arterial pressure tells healthcare practitioners about how effectively blood and oxygen are being delivered to tissue and organs (Handler, 2009). MAP is "the average pressure required that forces blood through the systemic organs" ((Ferns et al., $2010)$, pp. 41). It is a more accurate measurement in determining the actual pressure of blood against arterial walls (Handler, 2009). MAP should be used over SBP for the management of patients with acute conditions where there is a concern for appropriate organ perfusion (reference required). According to Bradshaw (2012), MAP has been used in hemodialysis as a guide to monitor intravascular changes and could also be beneficial in indicating critical changes in any patient acute or chronic to improve outcomes. The desirable range is $70-100 \mathrm{mmHg}$; a minimum of $60 \mathrm{mmHg}$ is required to supply enough blood to nourish the coronary arteries, brain and kidneys. If MAP falls below $60 \mathrm{mmHg}$ for an appreciable length of time, vital organs can be deprived of oxygen (Chapleau, 2012).

Pulse pressure is the difference between the systolic and diastolic $\mathrm{BP}$; it represents the force that the heart generates each time it contracts (Blood Pressure, 2020). For example, if the resting BP is $130 / 80 \mathrm{~mm} \mathrm{Hg}$, then the pulse pressure is $50 \mathrm{mmHg}$. A narrow pulse pressure can be an early indication of shock (Ferns et al., 2010). A narrowed pulse pressure is a cue for a clinician to suspect hypovolemia or a decrease in cardiac output.

\section{Serum creatinine as a measure of tissue perfusion}

The vital signs presented in Table 3 indicated that Mr. B had MAPs during his ED stay that likely were not adequate for effective tissue perfusion; inadequate perfusion limits the delivery of blood and oxygen to organs ultimately causing organ dysfunction and tissue death. Increased serum creatinine levels occur for many reasons, one of the most common being changes to kidney health and function. Prior to his visit, Mr. B had no known history of kidney dysfunction or failure. Mr. B's creatinine of $268 \mathrm{mmol} / \mathrm{L}$ could indicate acute renal failure. In retrospect, it is assumed that ineffective tissue perfusion due to hypovolemia and the resultant decreased MAP was a factor for Mr. B's acute kidney injury and failure. We believe that when put into context with the other abnormal lab values and the patient's presenting symptoms, the elevated serum creatinine was an early indicator of poor tissue perfusion caused by hypovolemia.

\section{Elevated serum lactate}

Lactate is a byproduct of carbohydrate metabolism and serum lactate rises as a result of increased cellular metabolism (LoCicero, 2018). Lactate levels are a reliable measure of 
tissue oxidation and a by-product of anaerobic metabolism. We can assume that increased metabolic demand and anaerobic metabolism can cause blood lactate levels to rise including most commonly in septicemia, but also in hemorrhage, myocardial infarction and pulmonary embolus. As indicated in Table 2, there was an upward trend in blood lactate levels when the patient decompensated. Mr. B's initial elevated blood lactate was treated with a fluid bolus, which may have affected his clotting cascade. Perhaps, if elevated lactate was discussed as a marker of poor oxidation, and not just as a benchmark of sepsis in our $\mathrm{ED}$, there would have been more investigation into his impaired tissue perfusion.

\section{The significance of elevated D-dimer}

Another confounding factor in Mr. B's presentation was his elevated serum $\mathrm{D}$-dimer levels coupled with his non-specific symptoms of shortness of breath and non-specific acute vs. chronic back pain. Nienaber \& Clough (2015) state "D-dimer is fibrin degradation product of a thrombus" (pp. 801). However, it is well known that $\mathrm{D}$-dimer elevation can occur with the presence of any thrombus, even a superficial contusion. Nienaber \& Clough (2015) indicate that in the case of AAA, D-dimer has a sensitivity of $97 \%$ and specificity of $47 \%$. Classically, an elevated D-dimer paired with a patient experiencing shortness of breath raises suspicion for a pulmonary embolus. However, Nienaber \& Clough (2015) suggest that D-dimers in significantly elevated levels are more likely in an acute aortic dissection than levels seen in patients with acute coronary syndromes, with a sensitivity of $98 \%$. As such, we believe that the significantly elevated D-dimer presented in this case should have led to further diagnostic workup before a diagnosis of acute aortic dissection was ruled out and the patient was treated with anticoagulants.

\section{A note on CTAS}

Upon presentation to the ED, Mr. B was triaged and assigned a CTAS score of 3. According to Bullard et al. (2017), Mr. B's initial abnormal vital signs support a CTAS 2 score. His report of chronic back pain may have been a factor in driving his score in the wrong direction. Furthermore, it is possible the lower CTAS score delayed Mr. B’s assessment by a physician.

\section{Education as a strategy to improve practice}

In recent years, a Canadian designed emergency nursing course has been developed to enhance decision-making within emergency nursing care. Emergency Practice Interventions \& Care Canada (EPICC), emphasizes the importance of assessment, monitoring and planning in emergency nursing (EPICC, 2018). Currently in our practice setting, a policy exists for hourly assessment of vital signs. This frequent vital sign assessment is further supported by nursing documentation forms designed for frequent charting and trending of vital signs including BP, pulse, RR \& SPO2. The form does not include a documentation area for recording and trending MAP. Educating ED nurses on MAP and including it on assessment and documentation records, supports improved monitoring and recognition of changes to hemodynamic status (EPICC, 2018). Furthermore, Chapleau (2012) proposes that monitoring MAP, as a means of determining adequate tissue perfusion, may help nurses identify and respond to acute changes in hemodynamic status. By trending and documenting changes in MAP, nurses may recognize life-threatening illness earlier. We believe that there is a need to further evaluate how MAP is used in other care settings particularly the ED to support inclusion of it as a standard of assessment in emergency and urgent care facilities across Canada.

\section{Teaching points}

1. By monitoring and trending MAP in patients that present with symptoms that indicate pulmonary/cardiac distress, and abnormal vital signs and lab values, earlier detection of hypovolemic shock and severe sepsis is possible. Nurses involved in caring for critically ill patients should be provided education on and encouraged to evaluate MAP as part of their regular practice.

2. Abdominal aortic aneurysm is a life-threatening emergency that may present with signs and symptoms similar to other acute problems such as pulmonary embolus, myocardial infarction and renal colic. Significantly elevated D-dimer lab values may be a sign of AAA and thus, this diagnosis should not be ruled out until advanced diagnostic imaging can be performed. Changes in serum creatinine and blood lactate should also be evaluated and considered as indicators of diminished tissue perfusion and not just indicators of one specific problem such as septicemia.

3. ED nurses care for critically ill patients and require a high level of education and orientation prior to entering their care setting. Standardized education and orientation to EDs should be developed nationwide to improve nursing practice and patient outcomes. Including EPICC foundations as part of new employee education in Canadian EDs is one strategy to provide new ER nurses with the tools to effectively assess, monitor and intervene in critical situations.

\section{Conclusion}

Mr. B's case reminds us that AAA is a serious condition with a high mortality rate. Often, these patients present to EDs with symptoms that warrant a wide differential diagnosis. When confounding factors exist such as non-specific symptoms, abnormal lab values that are relevant to AAA as well as other serious conditions, there is increased risk of missed diagnosis. As nurses, we can learn from our failure to recognize, assess, communicate or escalate when caring for a deteriorating patient. Broadening our assessment practice to include monitoring BP, MAP and PP may be one way to improve outcomes for our critically ill patients. Following the MAP may just save a life. Lastly, we identified the need for ongoing study and review of AAA patients in the ED as well as critical reflection and self-evaluation as essential component of emergency nursing practice.

\section{About the authors \\ Jennifer is an ER RN currently working in Lethbridge, $A B$. She graduated with Great Distinction from the University of Lethbridge's Bachelor of Nursing After Degree program. She has previous degrees in Exercise Science and Anthropology and has previously published work in the field of Exercise Physiology. She is currently studying for her MN. with a focus in shock physiology. When she's not nursing or studying, Jenn loves riding her Peloton}


spin bike, camping and hanging out with her husband, three daughters and two dogs.

Dawn lives in Lethbridge, Alberta and graduated from the RN program at the Lethbridge College in 1994 and obtained her Bachelor of Nursing at the University of Lethbridge in 2013. She is the current NENA-AB President and Director of Alberta with NENA-National. Dawn is also Chair of the International Advisory
Council with ENA. She has been nursing for 26 years, and in 2009 she started working in Emergency Nursing and found my home. Since 2014 Dawn has been the Clinical Instructor with AHS for the Rural South West Zone while still working casual in the CRH-ED. Dawn's interest are in advocacy for equal education and resources for all Emergency Nurses. She is the proud grandma of two beautiful little girls and enjoys spending time with them, hiking in the mountains, scrapbooking, reading and travelling.

\section{REFERENCES}

Badger, S., Bedenis, R., Blair, P. H., Ellis, P., Kee, F., \& Harkin, D. W. (2014). Endovascular treatment for ruptured abdominal aortic aneurysm. In The Cochrane Collaboration (Ed.), Cochrane Database of Systematic Reviews (p. CD005261.pub3). John Wiley \& Sons, Ltd. https://doi.org/10.1002/14651858.CD005261.pub3

Blood Pressure. (2020, March 12). Lippincott Advisor. Retrieved from http://advisor.lww.com/lna/document.do?bid=719792

Bradshaw, W. (2012). The importance of MAP as a patient assessment tool in hemodialysis and acute care. Australian Nursing Journal, 20(2), 26-29.

Bullard, M. J., Musgrave, E., Warren, D., Unger, B., Skeldon, T., Grierson, R., van der Linde, E., \& Swain, J. (2017). Revisions to the Canadian emergency department triage and acuity scale (CTAS) guidelines 2016. CJEM, 19(S2), S18-S27. https://doi. org/10.1017/cem.2017.365

Chapleau, M. W. (2012). Baroreceptor reflexes. In Robertson, D., Bagioni, I., Burnstock, G., Low, P. \& Paton, J. (Eds). Primer on the autonomic nervous system, 161-165. Academic Press.

Centers for Disease Control and Prevention. (2020). National Center for Health Statistics. Underlying Cause of Death 1999-2018 on CDC WONDER Online Database website. Retrieved from http://wonder.cdc.gov/ucd-icd10.html

Emergency Practice Intervention \& Care Canada (EPICC). (2018). About EPICC. Retrieved from https://epicclearning.ca/en/ about/
Ferns, T., Harris, G., McMahon, T., \& Wright, K. (2010). Mean arterial blood pressure and the assessment of acutely ill patients. Nursing Standard, 25(12), 40-44. https://doi.org/10.7748/ ns2010.11.25.12.40.c8113

Handler, J. (2009). The importance of accurate BP measurement. The Permanente Journal, 13(3), 51-54.

LoCicero, R. (2018). Lactate Dehydrogenase Test [Online]. Retrieved from https://www-nlm-nih-gov.ahs.idm.oclc.org/medlineplus/ ency/article/003471.htm

Mercer-Deadman, P (2014). Aortic dissections, aneurysms and ruptures: an emergency perspective. Canadian Journal of Emergency Nursing 37(1), 18-21.

Nienaber, C. \& Clough, R. E. (2015). Management of acute aortic dissection. The Lancet, 385(9970), 800-811. http://dx.doi. org/10.1016/s0140-6736(14)61005-9

Scheetz, L. (2006). Aortic dissection: This uncommon but dangerous cardiac condition can have pronounced symptoms. American Journal of Nursing, 106(4) 55-59. http://ovidsp.tx.ovid.com.ahs. idm.oclc.org/sp-3.38.0a/ovidweb.cgi

Woodrow, P. (2011). Abdominal aortic aneurysms: Clinical features, treatment and care. Nursing Standard, 25, 50-57.

Look for supplemental materials such as author interviews and podcasts at www.CJEN.ca

The Canadian Journal of Emergency Nursing (CJEN) is the Official Journal of the National Emergency Nurses Association (NENA) of Canada. This article has been made available at no cost in partnership with NENA and the University of Alberta Libraries. 